\title{
Article \\ Risk Factors of Inadequate Bowel Preparation for
Screening Colonoscopy
}

\author{
Efrat L. Amitay ${ }^{1, *}$, Tobias Niedermaier ${ }^{1}$, Anton Gies ${ }^{2}{ }^{-}$, Michael Hoffmeister ${ }^{1}$ and Hermann Brenner ${ }^{1,2,3}$ (I) \\ 1 Division of Clinical Epidemiology and Aging Research, German Cancer Research Center (DKFZ), \\ 69120 Heidelberg, Germany; t.niedermaier@dkfz-heidelberg.de (T.N.); \\ m.hoffmeister@dkfz-heidelberg.de (M.H.); h.brenner@dkfz.de (H.B.) \\ 2 Division of Preventive Oncology, German Cancer Research Center (DKFZ) and National Center for Tumor \\ Diseases (NCT), 69120 Heidelberg, Germany; anton.gies@nct-heidelberg.de \\ 3 German Cancer Consortium (DKTK), German Cancer Research Center (DKFZ), 69120 Heidelberg, Germany \\ * Correspondence: e.amitay@dkfz.de
}

Citation: Amitay, E.L.; Niedermaier, T.; Gies, A.; Hoffmeister, M.; Brenner, H. Risk Factors of Inadequate Bowel Preparation for Screening Colonoscopy. J. Clin. Med. 2021, 10, 2740. https://doi.org/10.3390/ jcm10122740

Academic Editor: Hiroyuki Yoshida

Received: 6 April 2021

Accepted: 17 June 2021

Published: 21 June 2021

Publisher's Note: MDPI stays neutral with regard to jurisdictional claims in published maps and institutional affiliations.

Copyright: () 2021 by the authors. Licensee MDPI, Basel, Switzerland. This article is an open access article distributed under the terms and conditions of the Creative Commons Attribution (CC BY) license (https:// creativecommons.org/licenses/by/ $4.0 /)$.

\begin{abstract}
The success of a colonoscopy in detecting and removing pre-cancerous and cancerous lesions depends heavily on the quality of bowel preparation. Despite efforts, $20-44 \%$ of colonoscopy participants have an inadequate bowel preparation. We aimed to assess and compare risk factors for inadequate bowel preparation and for the presence of advanced colorectal neoplasms in routine screening practice. In this cross-sectional study, among 8125 participants of screening colonoscopy in Germany with a comprehensive assessment of sociodemographic factors, lifestyle and medical history, we examined factors associated with inadequate bowel preparation and with findings of advanced neoplasms using adjusted log-binomial regression models. Among the identified risk factors assessed, three factors were identified that were significantly associated with inadequate bowel preparation: age $\geq 70$ years (adjusted prevalence ratios, aPR, 1.50 95\%CI 1.31-1.71), smoking (aPR 1.29 95\%CI 1.11-1.50) and abdominal symptoms (aPR 1.14 95\%CI 1.02-1.27). The same risk factors were also associated with the prevalence of advanced neoplasms in our study (aPR 1.72, 1.62 and 1.44, respectively). The risk factors associated with inadequate bowel preparation in this study were also associated with a higher risk for advanced neoplasms. Inadequate bowel preparation for colonoscopy might lead to missed colorectal cancer (CRC) precursors and the late diagnosis of CRC. People at high risk of advanced neoplasms are in particular need of enhanced bowel preparation.
\end{abstract}

Keywords: colorectal neoplasms; colonoscopy; cancer screening; bowel preparation

\section{Introduction}

Colorectal cancer (CRC) is the third most commonly diagnosed malignancy and the second most common cause of cancer-related death worldwide. In 2018, CRC accounted for over 1.8 million incident cases and almost 900,000 deaths [1].

Colonoscopy, conducted either for primary screening or as a follow-up for fecal occult blood tests, is the gold standard in early detection of CRC. Screening colonoscopy has the potential to prevent a very large share of CRC incidence and mortality by detecting and removing precursors of the disease [2-4]. The ability to detect precursors strongly depends on the quality of bowel preparation, which facilitates the clear visualization of the mucosal surface [5]. Despite efforts to facilitate the process, $20-44 \%$ of colonoscopy participants have been reported in previous studies to have inadequate bowel preparation [6-8].

Inadequate bowel preparation may lead to incomplete colonoscopies [9] and precancerous lesions being missed and may necessitate repeat colonoscopy exams, a burden both to patients and the health system. The ability to identify factors associated with inadequate bowel preparation may help in targeting efforts to increase adequate preparation towards populations at higher risk. Therefore, we aimed to assess factors associated with the quality 
of bowel preparation for colonoscopy in a large routine screening colonoscopy cohort in Germany.

\section{Materials and Methods}

Our analysis is based on participants from KolosSal, a large German CRC screening cohort which has been described in detail elsewhere [10]. Briefly, the primary aim of KolosSal was monitoring the long-term reduction in CRC incidence and mortality among participants of screening colonoscopy, which has been offered in Germany since 2002. Overall, 19,177 participants of screening colonoscopy were recruited in gastroenterological practices in the German state of Saarland during 2005-2013 and completed a short questionnaire. Information on the quality of bowel preparation and findings at colonoscopy were extracted from colonoscopy records. The study was approved by the ethics committees of the medical faculty of the Heidelberg University and the Saarland Medical Association, and written informed consent was obtained from each participant.

The current analysis was restricted to 8125 participants with no colonoscopy in the previous year for whom the quality of bowel preparation was explicitly reported in the colonoscopy record ( $n=279$ participants with a previous colonoscopy in the past year were excluded). Information on bowel preparation and findings at colonoscopy were reported by clinicians as free text and extracted in a standardized manner. Whenever the report included remarks on impaired cleanliness of the bowel, i.e., impaired visibility or obstruction due to stool, or an incomplete colonoscopy due to insufficient bowel cleanliness, bowel preparation was classified as inadequate.

Based on such information, the quality of bowel preparation was classified as adequate or inadequate. Participants with adequate and inadequate bowel preparation were compared with respect to factors known or suspected to be associated with CRC risk: sex, age at colonoscopy, body mass index (BMI), education, smoking, alcohol consumption, red and processed meat consumption, aspirin use, past large bowel endoscopy, hypertension, diabetes and abdominal symptoms prior to colonoscopy. Log-binomial regression was used to quantify the independent association of those factors with (i) the quality of bowel preparation and (ii) the detection of at least one advanced neoplasm (CRC or advanced adenomas; thus, defined if they matched any of the following criteria: size $\geq 1 \mathrm{~cm}$, villous or tubulo-villous architecture or high-grade dysplasia). The analysis of associations with findings of advanced neoplasms was restricted to those with reported adequate bowel preparation to minimize potential bias by missed neoplasms.

Analyses were conducted in R 3.6.1 [11] and ten multiple imputations were performed using the "mice" package to deal with missing values in exposure variables $(0-16 \%$ missing). Statistical significance was defined by a $p$-value of $p<0.05$ in 2-sided testing.

\section{Results}

Table 1 shows main characteristics of the study population and their associations with the quality of bowel preparation. Overall, 8125 study participants were eligible for analysis. Women accounted for $49.5 \%$ of participants and the mean age was $63.3 \pm 6.9$ years. Slightly more than half of the participants $(52.1 \%)$ smoked in the past or were smoking at the time of joining the study and for $70.4 \%$ it was their first colonoscopy.

As seen in Table 1, inadequate bowel preparation was significantly more often reported for participants $\geq 70$ years of age $(22.5 \%)$, current smokers $(20.4 \%)$, regular users of aspirin $(19.5 \%)$, participants with diabetes $(20.5 \%)$ and participants who reported abdominal symptoms before colonoscopy (19.9\%). In multivariate adjusted analyses, statistically significant prevalence ratios (aPRs, $95 \% \mathrm{CI}$ ) were found for participants $\geq 70$ years of age $(\mathrm{aPR}=1.50,95 \% \mathrm{CI} 1.31-1.71)$, current smokers $(\mathrm{aPR}=1.29,95 \% \mathrm{CI} 1.11-1.50)$ and those reporting abdominal symptoms before colonoscopy (aPR $=1.14,95 \% \mathrm{CI} 1.02-1.27$ ). 
Table 1. Characteristics of the study population, association with inadequate bowel preparation and risk of advanced neoplasms.

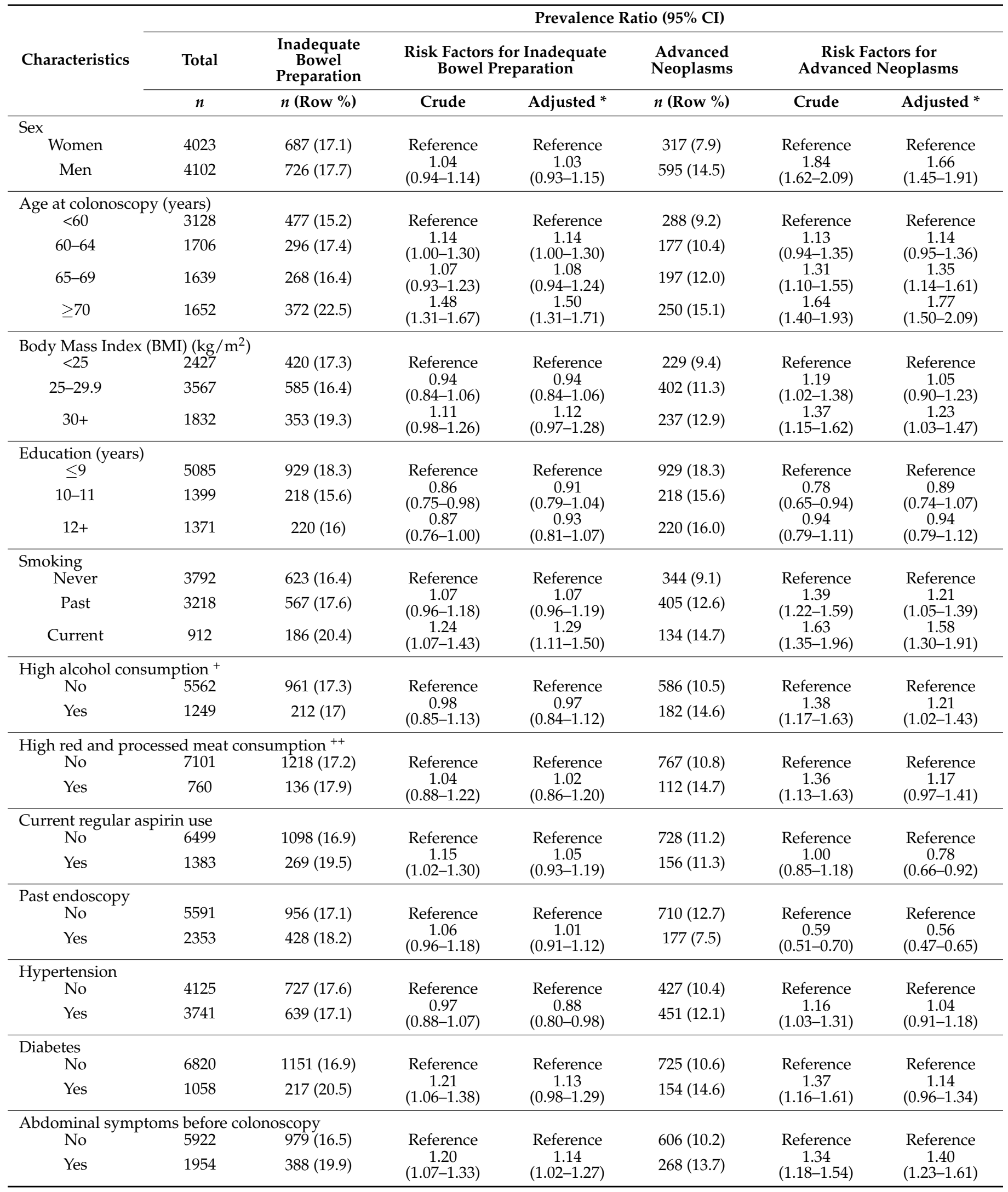

* Adjusted for all other covariates listed in the column; ${ }^{+}$High alcohol consumption: regular intake of alcohol on $\geq 5$ days a week with one standard alcoholic beverage equaling $0.33 \mathrm{~L}$ beer, $0.25 \mathrm{~L}$ wine or $2 \mathrm{cL}$ hard liquor. ${ }^{++}$Defined as high if the participant consumed red and processed meat at least once per day. Abbreviations: CI—confidence interval; col\%—column percent, e.g., 4023/ $(4023+4102)=49.5 \%$ for women in variable "sex"; Row\%—row percent, e.g., $687 / 4023=17.1 \%$ for inadequate bowel preparation among women. 
Table 2 presents the risk factors for advanced neoplasms among study participants with adequate bowel preparation. These same factors, which were found to be associated with inadequate bowel preparation, were also found to be strongly associated with findings of advanced neoplasms among those with adequate bowel preparation, with aPR $=1.72$ (95\%CI 1.42-2.08), aPR = 1.62 (95\%CI 1.31-2.00) and $\mathrm{aPR}=1.44$ (95\%CI 1.24-1.68), for age $\geq 70$ years, current smoking and abdominal symptoms, respectively.

Table 2. Characteristics of the study population and association with prevalence of advanced neoplasms among participants with adequate bowel preparation.

\begin{tabular}{|c|c|c|c|c|c|c|}
\hline \multirow{3}{*}{ Characteristics } & \multicolumn{4}{|c|}{ Participants } & \multicolumn{2}{|c|}{ Prevalence Ratio (95\% CI) } \\
\hline & \multicolumn{2}{|c|}{ Adequate Bowel Preparation } & \multicolumn{2}{|c|}{ Advanced Neoplasms } & \multirow{2}{*}{ Crude } & \multirow{2}{*}{ Adjusted * } \\
\hline & $n$ & Col \% & $n$ & Row \% & & \\
\hline \multicolumn{7}{|l|}{ Sex } \\
\hline Women & 3336 & 49.7 & 260 & 7.8 & Reference & Reference \\
\hline Men & 3376 & 50.3 & 481 & 14.2 & $1.83(1.58-2.11)$ & $1.65(1.41-1.92)$ \\
\hline \multicolumn{7}{|c|}{ Age at colonoscopy (years) } \\
\hline$<60$ & 2651 & 39.5 & 244 & 9.2 & Reference & Reference \\
\hline $60-64$ & 1410 & 21.0 & 152 & 10.8 & $1.17(0.97-1.42)$ & $1.20(0.99-1.45)$ \\
\hline $65-69$ & 1371 & 20.4 & 162 & 11.8 & $1.28(1.06-1.55)$ & $1.34(1.11-1.62)$ \\
\hline$\geq 70$ & 1280 & 19.1 & 183 & 14.3 & $1.55(1.30-1.86)$ & $1.72(1.42-2.08)$ \\
\hline \multicolumn{7}{|c|}{ Body Mass Index (BMI) $\left(\mathrm{kg} / \mathrm{m}^{2}\right)$} \\
\hline$<25$ & 2007 & 31.0 & 187 & 9.3 & Reference & Reference \\
\hline $25-29.9$ & 2982 & 46.1 & 330 & 11.1 & 1.19 (1.00-1.41) & $1.06(0.89-1.26)$ \\
\hline $30+$ & 1479 & 22.9 & 186 & 12.6 & $1.36(1.13-1.65)$ & $1.26(1.03-1.54)$ \\
\hline \multicolumn{7}{|l|}{ Education (years) } \\
\hline$\leq 9$ & 4156 & 64.1 & 475 & 11.4 & Reference & Reference \\
\hline $10-11$ & 1181 & 18.2 & 104 & 8.8 & $0.77(0.63-0.94)$ & $0.86(0.71-1.05)$ \\
\hline $12+$ & 1151 & 17.7 & 132 & 11.5 & $0.99(0.83-1.19)$ & $1.00(0.83-1.20)$ \\
\hline \multicolumn{7}{|l|}{ Smoking } \\
\hline Never & 3169 & 48.4 & 280 & 8.8 & Reference & Reference \\
\hline Past & 2651 & 40.5 & 326 & 12.3 & $1.39(1.19-1.61)$ & $1.20(1.03-1.41)$ \\
\hline Current & 726 & 11.1 & 109 & 15.0 & $1.70(1.38-2.09)$ & $1.62(1.31-2.00)$ \\
\hline \multicolumn{7}{|c|}{ High alcohol consumption $^{+}$} \\
\hline No & 4601 & 81.6 & 473 & 10.3 & Reference & Reference \\
\hline Yes & 1037 & 18.4 & 151 & 14.6 & $1.42(1.19-1.69)$ & $1.24(1.04-1.48)$ \\
\hline \multicolumn{7}{|c|}{ Red and processed meat consumption } \\
\hline$<1$ per day & 5883 & 90.4 & 626 & 10.6 & Reference & Reference \\
\hline$\geq 1$ per day & 624 & 9.6 & 89 & 14.3 & $1.34(1.09-1.64)$ & $1.16(0.95-1.42)$ \\
\hline \multicolumn{7}{|c|}{ Current regular aspirin use } \\
\hline No & 5401 & 82.9 & 598 & 11.1 & Reference & Reference \\
\hline Yes & 1114 & 17.1 & 119 & 10.7 & $0.96(0.80-1.16)$ & $0.79(0.65-0.95)$ \\
\hline \multicolumn{7}{|l|}{ Past endoscopy } \\
\hline No & 4635 & 70.7 & 579 & 12.5 & Reference & Reference \\
\hline Yes & 1925 & 29.3 & 140 & 7.3 & $0.58(0.49-0.70)$ & $0.55(0.46-0.65)$ \\
\hline \multicolumn{7}{|l|}{ Hypertension } \\
\hline No & 3398 & 52.3 & 352 & 10.4 & Reference & Reference \\
\hline Yes & 3102 & 47.7 & 362 & 11.7 & $1.12(0.97-1.29)$ & $1.01(0.87-1.17)$ \\
\hline \multicolumn{7}{|l|}{ Diabetes } \\
\hline No & 5669 & 87.1 & 598 & 10.5 & Reference & Reference \\
\hline Yes & 841 & 12.9 & 117 & 13.9 & $1.32(1.10-1.59)$ & $1.10(0.91-1.33)$ \\
\hline \multicolumn{7}{|c|}{ Abdominal symptoms before colonoscopy } \\
\hline No & 4943 & 75.9 & 496 & 10.0 & Reference & Reference \\
\hline Yes & 1566 & 24.1 & 212 & 13.5 & $1.36(1.17-1.58)$ & $1.44(1.24-1.68)$ \\
\hline
\end{tabular}

${ }^{*}$ Adjusted for all other covariates listed in the column; ${ }^{+}$High alcohol consumption: regular intake of alcohol on $\geq 5$ days a week with one standard alcoholic beverage equaling $0.33 \mathrm{~L}$ beer, $0.25 \mathrm{~L}$ wine or $2 \mathrm{cL}$ hard liquor. Abbreviations: CI-confidence interval; col\%—column percent, e.g., $4023 /(4023+4102)=49.5 \%$ for women in variable "sex"; Row $\%$-row percent, e.g., 687/4023 = 17.1\% for inadequate bowel preparation among women. 


\section{Discussion}

In this study, among more than 8000 participants of screening colonoscopy, some of the strongest risk factors for the presence of CRC, i.e., old age, smoking and abdominal symptoms, were suggested to also be risk factors for inadequate bowel preparation. Since poor bowel preparation goes along with an increased risk of missed relevant colorectal neoplasms [9] these results underline the importance of efforts to ensure the best possible bowel preparation in routine screening practice.

A number of previous, mostly smaller, studies have assessed the prevalence and risk factors of poor bowel preparation in screening and clinical settings as shown in Supplementary Table S1 [8,12-15]. The association of inadequate bowel preparation with older age may be explained by a worse mental and functional state. In one study, failed colonoscopy among nonagenarians was due to a bad preparation which was inversely associated with the mental and functional state [16]. Abdominal symptoms may be due to anatomical reason or medical disorders that lead to constipation or obstructions in the bowel which were found to be associated with inadequate bowel preparation due to infrequent bowel movements [17].

To our knowledge, ours is the largest study that investigated the role of risk factors for poor bowel preparation exclusively in an outpatient routine screening setting, and the first one to asses several risk factors of inadequate bowel preparation and of advanced colorectal neoplasms side by side in the same study population. In order to minimize bias from missed advanced neoplasms, the latter association was assessed among participants for whom adequate bowel preparation was explicitly documented.

Our results are based on a questionnaire and colonoscopy data collected from participants of a German population-based CRC screening colonoscopy program. With 8125 participants, it is the largest study to date to investigate factors associated with inadequate bowel preparation for colonoscopy conducted solely in a population screening setting. Since adequate bowel cleaning and completed colonoscopy rates differ between inpatient and screening setting [18], our study offers a more accurate view on groups that may need more guidance in preparing for colonoscopies in a population screening setting.

Our study also has limitations. The study included participants who not only chose to attend screening colonoscopy, but also to participate in the study. Most likely populations that tend to have lower participation rates in screening programs such as those with language barriers or from lower socio-economic backgrounds will be underrepresented [19]. Possibly, these groups might also have greater difficulties in performing bowel cleaning properly according to instructions; thus, possibly leading to an even higher rate of inadequate bowel preparation. The information is based on self-administered questionnaires.

Another limitation of our study is that the quality of bowel preparation was reported in approximately half of the colonoscopy reports only and was not rated by an established score. The latter may have led to an imperfect classification of the quality of bowel preparation and, as a result, to some underestimation of associations. On the other hand, our results may well reflect routine screening practice outside academic centers where most screening colonoscopies are conducted and standardized documentation of the quality of bowel preparation is not established.

In conclusion, our study suggests that risk factors for advanced neoplasms such as older age, smoking and having ongoing abdominal symptoms are also risk factors for inadequate bowel preparation for colonoscopy, possibly leading to missed CRC precursors during screening colonoscopy. More efforts should be made to better identify and prepare these higher risk populations adequately for screening colonoscopies.

Supplementary Materials: The following are available online at https://www.mdpi.com/article/10 $.3390 / \mathrm{jcm} 10122740 / \mathrm{s} 1$, Table S1: Previous studies assessing risk factors for inadequate bowel preparation.

Author Contributions: Conceptualization, H.B.; Formal analysis, E.L.A.; Funding acquisition, M.H. and H.B.; Methodology, E.L.A., T.N., M.H. and H.B.; Writing—original draft, E.L.A.; Writing—review 
and editing, E.L.A., T.N., A.G., M.H. and H.B. All authors have read and agreed to the published version of the manuscript.

Funding: This study was supported in part by grants from the Central Research Institute of Ambulatory Health Care in Germany, Berlin, Germany, and from the German Cancer Aid (nos. 108230, 110553). The sponsors had no role in the study design and in the collection, analysis, and interpretation of data.

Institutional Review Board Statement: The KolosSal study was approved by ethics committees of the University of Heidelberg (057/2005) and of the Medical Association of Saarland (54/05).

Informed Consent Statement: Informed consent was obtained from all subjects involved in the study.

Data Availability Statement: The data presented in this study are available on request from the corresponding author. The data are not publicly available due to privacy restrictions.

Acknowledgments: We are grateful to the physicians conducting screening colonoscopies for their excellent cooperation in patient recruitment, and to all colleagues in the study center for data collection and cleaning, monitoring and documentation.

Conflicts of Interest: The authors declare no conflict of interest. The funders had no role in the design of the study; in the collection, analyses, or interpretation of data; in the writing of the manuscript, or in the decision to publish the results.

\section{References}

1. Bray, F.; Ferlay, J.; Soerjomataram, I.; Siegel, R.L.; Torre, L.A.; Jemal, A. Global cancer statistics 2018: GLOBOCAN estimates of incidence and mortality worldwide for 36 cancers in 185 countries. CA Cancer J. Clin. 2018, 68, 394-424. [CrossRef]

2. Brenner, H.; Stock, C.; Hoffmeister, M. Effect of screening sigmoidoscopy and screening colonoscopy on colorectal cancer incidence and mortality: Systematic review and meta-analysis of randomised controlled trials and observational studies. BMJ 2014, 348, g2467. [CrossRef]

3. Chen, C.; Stock, C.; Hoffmeister, M.; Brenner, H. Public health impact of colonoscopy use on colorectal cancer mortality in Germany and the United States. Gastrointest. Endosc. 2018, 87, 213-221.e2. [CrossRef] [PubMed]

4. Brenner, H.; Chen, C. The colorectal cancer epidemic: Challenges and opportunities for primary, secondary and tertiary prevention. Br. J. Cancer 2018, 119, 785-792. [CrossRef] [PubMed]

5. Sulz, M.C.; Kröger, A.; Prakash, M.; Manser, C.N.; Heinrich, H.; Misselwitz, B. Meta-Analysis of the Effect of Bowel Preparation on Adenoma Detection: Early Adenomas Affected Stronger than Advanced Adenomas. PLoS ONE 2016, 11, e0154149. [CrossRef] [PubMed]

6. Adler, A.; Wegscheider, K.; Lieberman, D.; Aminalai, A.; Aschenbeck, J.; Drossel, R.; Mayr, M.; Mroß, M.; Scheel, M.; Schröder, A.; et al. Factors determining the quality of screening colonoscopy: A prospective study on adenoma detection rates, from 12134 examinations (Berlin colonoscopy project 3, BECOP-3). Gut 2013, 62, 236-241. [CrossRef] [PubMed]

7. Froehlich, F.; Wietlisbach, V.; Gonvers, J.-J.; Burnand, B.; Vader, J.-P. Impact of colonic cleansing on quality and diagnostic yield of colonoscopy: The European Panel of Appropriateness of Gastrointestinal Endoscopy European multicenter study. Gastrointest. Endosc. 2005, 61, 378-384. [CrossRef]

8. Fayad, N.F.; Kahi, C.J.; El-Jawad, K.H.A.; Shin, A.S.; Shah, S.; Lane, K.A.; Imperiale, T. Association Between Body Mass Index and Quality of Split Bowel Preparation. Clin. Gastroenterol. Hepatol. 2013, 11, 1478-1485. [CrossRef]

9. Chokshi, R.V.; Hovis, C.E.; Hollander, T.; Early, D.S.; Wang, J.S. Prevalence of missed adenomas in patients with inadequate bowel preparation on screening colonoscopy. Gastrointest. Endosc. 2012, 75, 1197-1203. [CrossRef] [PubMed]

10. Brenner, H.; Haug, U.; Arndt, V.; Stegmaier, C.; Altenhofen, L.; Hoffmeister, M. Low Risk of Colorectal Cancer and Advanced Adenomas More Than 10 Years After Negative Colonoscopy. Gastroenterology 2010, 138, 870-876. [CrossRef]

11. R Core Team. R: A Language and Environment for Statistical Computing; R Foundation for Statistical Computing: Vienna, Austria, 2019. Available online: http:/ / www.R-project.org/ (accessed on 6 April 2021).

12. Hyun, J.H.; Kim, S.J.; Park, J.H.; Wie, G.A.; Kim, J.-S.; Han, K.S.; Kim, B.C.; Hong, C.W.; Sohn, D.K. Lifestyle Factors and Bowel Preparation for Screening Colonoscopy. Ann. Coloproctol. 2018, 34, 197-205. [CrossRef]

13. Hassan, C.; Fuccio, L.; Bruno, M.; Pagano, N.; Spada, C.; Carrara, S.; Giordanino, C.; Rondonotti, E.; Curcio, G.; Dulbecco, P.; et al. A Predictive Model Identifies Patients Most Likely to Have Inadequate Bowel Preparation for Colonoscopy. Clin. Gastroenterol. Hepatol. 2012, 10, 501-506. [CrossRef] [PubMed]

14. Lebwohl, B.; Wang, T.C.; Neugut, A.I. Socioeconomic and Other Predictors of Colonoscopy Preparation Quality. Dig. Dis. Sci. 2010, 55, 2014-2020. [CrossRef] [PubMed]

15. Borg, B.B.; Gupta, N.; Zuckerman, G.R.; Banerjee, B.; Gyawali, C.P. Impact of Obesity on Bowel Preparation for Colonoscopy. Clin. Gastroenterol. Hepatol. 2009, 7, 670-675. [CrossRef] [PubMed]

16. Schmilovitz-Weiss, H.; Weiss, A.; Boaz, M.; Levin, I.; Chervinski, A.; Shemesh, E. Predictors of failed colonoscopy in nonagenarians: A single-center experience. J. Clin. Gastroenterol. 2007, 41, 388-393. [CrossRef] [PubMed] 
17. Lee, D.-W.; Koo, J.S.; Kang, S.; Kim, S.Y.; Hyun, J.J.; Jung, S.W.; Yim, H.J.; Lee, S.W. Association between bowel habits and quality of bowel preparation for colonoscopy. Medicine 2017, 96, e7319. [CrossRef] [PubMed]

18. Hendry, P.O.; Jenkins, J.T.; Diament, R.H. The impact of poor bowel preparation on colonoscopy: A prospective single centre study of 10571 colonoscopies. Color. Dis. 2007, 9, 745-748. [CrossRef] [PubMed]

19. Smith, S.; McGregor, L.; Raine, R.; Wardle, J.; Von Wagner, C.; Robb, K. Inequalities in cancer screening participation: Examining differences in perceived benefits and barriers. Psycho Oncol. 2016, 25, 1168-1174. [CrossRef] [PubMed] 